\title{
Knockdown of RAP2A gene expression suppresses cisplatin resistance in gastric cancer cells
}

\author{
JINYU ZHANG ${ }^{1}$, YUNHAI WEI ${ }^{1}$, JIE MIN ${ }^{2}$, YAN WANG $^{1}$, LEI YIN ${ }^{1}$, GUOLIANG CAO $^{1}$ and HUA SHEN ${ }^{1}$ \\ ${ }^{1}$ Department of Gastrointestinal Surgery; ${ }^{2}$ Intensive Care Unit, Huzhou Central Hospital, \\ Affiliated Central Hospital Huzhou University, Huzhou, Zhejiang 313000, P.R. China
}

Received September 29, 2018; Accepted July 9, 2019

DOI: $10.3892 / \mathrm{ol} .2019 .11086$

\begin{abstract}
Cisplatin (DDP) resistance is closely associated with the failure of chemotherapy to manage various different types of human cancer. The GTPase protein Ras-related protein Rap-2a (RAP2A) regulates cancer cell proliferation, migration and invasion; however, little is currently known regarding its role in cancer cell resistance to chemotherapy. The present study investigated the potential roles of the RAP2A gene in gastric cancer cell resistance to DDP treatment. The DDP half maximal inhibitory concentration $\left(\mathrm{IC}_{50}\right)$ values for the proliferation inhibition of MGC803 and MGC803/DDP gastric cancer cells were determined by treating the cells with a DDP concentration gradient and measuring their survival rates using the Cell Counting Kit- 8 (CCK-8) assay; cell viability was also assessed using the CCK-8 assay. Cell migration and invasion were assessed using Transwell Matrigel assays, and apoptosis and DNA damage were evaluated using flow cytometry and Hoechst staining. RAP2A expression was knocked down by siRNA transfection, and RAP2A protein levels were examined using western blotting. The DDP $\mathrm{IC}_{50}$ values for DDP-resistant MGC803/DDP cells were greater than those for MGC803 cells. Furthermore, MGC803/DDP cells exhibited increased levels of viability, migration and invasion, and decreased levels of apoptosis and DNA damage during DDP treatment. Knockdown of RAP2A expression significantly promoted MGC803/DDP cell apoptosis and DNA damage, and decreased the viability and invasion capabilities of these cells following treatment with DDP. The results of the present study revealed that RAP2A expression promotes DDP resistance in gastric cancer cells by increasing their viability, migration and invasion capacities, and by suppressing apoptosis and DNA damage.
\end{abstract}

Correspondence to: Professor Hua Shen, Department of Gastrointestinal Surgery, Huzhou Central Hospital, Affiliated Central Hospital Huzhou University, 198 Hongqi Road, Huzhou, Zhejiang 313000, P.R. China

E-mail:drminjie@163.com

Key words: Ras-related protein Rap-2a, gastric cancer, cisplatin resistance, migration and invasion, apoptosis, DNA damage

\section{Introduction}

Gastric cancer is a severe malignant disease that develops in the lining of the stomach, and is the third leading cause of cancer-associated mortality worldwide (1). In 2012, there were $~ 951,600$ cases of gastric cancer and 723,100 deaths (2). During the later stages of disease, patients with gastric cancer usually display severe symptoms, such as upper abdominal pain, weight loss and difficulty swallowing, which results from metastasis to other organs, such as the lymph nodes, liver and lungs (3). Previous epidemiological studies demonstrated that gastric cancer development has been associated with a number of causative factors, including Helicobacter pylori infection, cigarette smoking, dietary habits and genetic mutations, as well as pathogenic conditions such as pernicious anemia, diabetes and chronic atrophic gastritis $(4,5)$. Among the causative factors, chronic infections induced by the bacterium Helicobacter pylori have been established as the most common cause of gastric cancer, and are responsible for $\sim 90 \%$ of noncardia gastric cancer worldwide (6). Due to a lack of specific symptoms during the early stages of disease, gastric cancer is often diagnosed at an advanced stage, and this late diagnosis is the primary reason for the poor prognosis observed in the majority of patients (7). There is an urgent requirement for the development of new diagnostic methods and novel therapeutics to decrease gastric cancer-associated mortality and improve the clinical outcomes of patients.

Currently, the primary methods used to treat gastric cancer are surgery, chemotherapy and radiotherapy (8-10). The only known curative therapies for gastric cancer are surgical procedures such as endoscopic mucosal resection and endoscopic submucosal dissection (11); however, these methods are only suitable for patients with early-stage gastric cancer. Chemotherapy, radiotherapy and newly developed targeted therapies have primarily been used to treat patients with later stage disease or those where the cancer has metastasized to other organs $(10,12,13)$. In addition, chemotherapy has been used to shrink gastric tumors prior to surgery, or to eradicate any remaining cancerous cells following surgery (10). A number of different chemotherapeutic agents have been used in the treatment of gastric cancer, including fluorouracil, carmustine, doxorubicin, mitomycin $\mathrm{C}$, taxotere and cisplatin (DDP) $(10,14)$. DDP is one of the chemotherapy agents most widely used to treat number of different types of cancer, but its use is limited by the occurrence of multiple side effects and the frequent development of resistance (15). 
DDP resistance has been associated with changes in its cellular uptake and efflux, increased DNA repair efficiency, decreased rates of cell apoptosis and increased cellular detoxification activity $(15,16)$. A number of reports have provided new insights into the molecular processes that mediate DDP resistance in gastric cancer cells; microRNA (miR)-21 was demonstrated to promote DDP resistance in gastric cancer cells by suppressing the expression of the phosphatase and tension homolog deleted on chromosome 10 gene and activating the protein kinase B (AKT) signaling pathway (17). Furthermore, AKT signaling cascades, together with hypoxia-inducible factor $1 \alpha$, may enhance the expression of the survivin gene, which contributes to the development of DDP resistance in gastric cancer cells (18). Other molecular factors that may contribute to DDP resistance in these cells include miR-1271 (19), X-ray repair cross complementing group 1, thioredoxin-like protein 1 (20) and numerous other functional proteins associated with cell proliferation and apoptosis. However, the mechanisms of DDP resistance in gastric cancer cells are yet to be fully elucidated.

Ras-related protein Rap-2A (RAP2A), is a member of the small GTPase protein superfamily and a target of the p53 transcription factor, which is associated with multiple cellular processes including cell proliferation, adhesion and migration $(21,22)$. Furthermore, RAP2A was demonstrated to promote cancer cell migration, invasion and metastasis by activating the AKT signaling pathway $(21,22)$. However, the role of RAP2A in the development of cellular resistance to chemotherapy remains largely unknown. In the present study, the potential roles of RAP2A in regulating the induced resistance of gastric cancer cells to DDP were investigated, with the aim of gaining new insights into the molecular mechanisms underlying chemotherapy resistance.

\section{Materials and methods}

Cell culture and reagents. The human gastric cancer cell line MGC803 (BNCC100665) was purchased from the BeNa Culture Collection and cultured in RPMI-1640 medium (Thermo Fisher Scientific, Inc.) supplemented with $10 \%$ fetal bovine serum (FBS; cat. no. 26140079; Gibco; Thermo Fisher Scientific, Inc.), at $37^{\circ} \mathrm{C}$ in a humidified atmosphere $\left(5 \% \mathrm{CO}_{2}\right)$. MGC803/DDP cells with induced resistance to cisplatin (DDP; cat. no. 15663-27-1; Sigma-Aldrich; Merck KGaA) were obtained by exposing MGC803 cells to a concentration gradient of DDP as previously described (23).

RAP2A knockdown. In order to suppress RAP2A expression, cultured gastric cancer cells were transfected with RAP2A small interfering (si)RNA. The siRNAs targeting the RAP2A gene were designed using the Whitehead Institute Web Server (http://jura.wi.mit.edu/bioc/siRNAext) and synthesized by Sangon Biotech Co., Ltd. An additional non-targeting scrambled siRNA served as a negative control (NC) was also designed and synthesized by Sangon Biotech Co., Ltd. The sequences of the RAP2A and NC siRNAs were as follows: RAP2A SiRNA \#1, 5'-AUACUUCUCUCUCACUUUCCA-3'; RAP2A siRNA \#2, 5'-ACUCUUAGCGGA AGUUUC CAU-3'; RAP2A siRNA \#3, 5'-UCGUAUUUCUCGAUG AAGGUG-3'; NC siRNA, 5'-UUCGUCUGUACUCCACAU ATT-3'. Gastric cancer cells were transfected with the siRNAs using Lipofectamine ${ }^{\circledR} 2000$ transfection reagent (Invitrogen; Thermo Fisher Scientific, Inc.) according to the manufacturer's protocol. At 2-7 days post-transfection, the levels of RAP2A protein expression were determined using western blot analyses, and further cellular assays were subsequently conducted. All experiments were independently repeated $\geq 3$ times.

Cell Counting Kit-8 (CCK-8) assay and determination of the half maximal inhibitory concentration $\left(I C_{50}\right)$ of DDP. Cell viability was analyzed using the $\mathrm{CCK}-8$ assay (Beyotime Institute of Biotechnology) according to the manufacturer's protocol. For the determination of the $\mathrm{IC}_{50}$ value of DDP, MGC803 and MGC803/DDP cells (3,000 cells/well) were seeded into 96-well plates and treated with a DDP concentration gradient of $0,0.5,1,2,4,6,8$ and $10 \mu \mathrm{g} / \mathrm{ml}$ for $24 \mathrm{~h}$. After cultivation in RPMI-1640 medium (Thermo Fisher Scientific, Inc.) with $10 \%$ FBS (cat. no. 26140079; Gibco; Thermo Fisher Scientific, Inc.) at $37^{\circ} \mathrm{C}$ in a humidified incubator with $5 \%$ $\mathrm{CO}_{2}$ for $24 \mathrm{~h}, \mathrm{CCK}-8$ solution was added to each well and subsequently incubated at the same culture conditions for $3 \mathrm{~h}$. The absorbance of each well at $450 \mathrm{~nm}\left(\mathrm{OD}_{450}\right)$ was measured with a microplate reader, and SPSS software (version 18.0; SPSS Inc.) was used to calculate the $\mathrm{IC}_{50}$ value. In order to assess the influence of DDP on cell viability, MGC803 and MGC803/DDP cells were treated with $2 \mu \mathrm{g} / \mathrm{ml}$ DDP for 24,48 and $72 \mathrm{~h}$, respectively, after which their viability was assessed using the aforementioned CCK-8 method.

Cell migration and invasion. The migration and invasion capabilities of gastric cancer cells treated with DDP and/or RAP2A siRNAs were determined using the Transwell system (Corning Inc.) as previously described (24), but with minor modifications. For the migration assay, the treated MGC803 cells were starved overnight in serum free medium then seeded $\left(200 \mu \mathrm{l} ; 1 \times 10^{5}\right.$ cells $\left./ \mathrm{ml}\right)$ in the upper chamber of the Transwell system, and $500 \mu$ l RPMI-1640 medium (Thermo Fisher Scientific, Inc.) with 15\% FBS (cat. no. 26140079; Gibco; Thermo Fisher Scientific, Inc.) was plated in the lower chamber. After cultivation at $37^{\circ} \mathrm{C}$ with $5 \% \mathrm{CO} 2$ for $24 \mathrm{~h}$, the cells were then allowed to vertically migrate through the membrane and into the lower chamber. The migrated cells in the lower chamber were fixed with $4 \%$ paraformaldehyde (cat. no. P1110; Beijing Solarbio Science and Technology Co., Ltd.) at room temperature for $10 \mathrm{~min}$ and stained with $0.5 \%$ crystal violet (cat. no. C-6158; Sigma-Aldrich; Merck KGaA) at room temperature for $5 \mathrm{~min}$. The results were carefully counted under an inverted light microscope (Olympus IX 73; Olympus Corporation) at x100 magnification. For the analysis of invasion capability, the treated MGC803 cells in serum-free medium were seeded $\left(1 \times 10^{5}\right.$ cells $\left./ \mathrm{ml}\right)$ into the upper chambers of a Corning Transwell system coated with Matrigel. Invaded cells were also stained with crystal violet (5\%) at room temperature for $5 \mathrm{~min}$. The remaining steps were the same as the migration assay. The numbers of cells in $\leq 8$ randomly selected visual fields were counted and each experiment was performed in triplicate.

Cell apoptosis assay. The apoptotic rates of gastric cancer cells were determined via flow cytometry using the Dead 
Cell Apoptosis kit (Thermo Fisher Scientific, Inc.), containing Annexin V FITC and propidium iodide (PI) according to the manufacturer's protocol. Following treatment with DDP, gastric cancer cells were washed three times in PBS solution and resuspended in Annexin-binding buffer, and subsequently incubated with FITC Annexin V and PI solution for 14 min at room temperature. The cells were incubated in $300 \mu \mathrm{l}$ Annexin-binding buffer, and the numbers of apoptotic cells were determined by a FACS Calibur flow cytometry (BD Biosciences). The apoptosis rate was quantitatively analyzed using BD CellQuest software version 3.3 (BD Biosciences). The percentage of apoptotic cells was determined for at $\leq 3$ independent experiments.

DNA damage assay. As previously described (25), the degree of DNA damage in the gastric cancer cells was determined by staining with Hoechst 33342 and DAPI (both Thermo Fisher Scientific, Inc.). Briefly, the treated cells were fixed with $4 \%$ paraformaldehyde (cat. no. P1110; Beijing Solarbio Science and Technology Co., Ltd.) at room temperature for $20 \mathrm{~min}$, and then stained with Hoechst 33342 Working Solution at room temperature in the dark for $10 \mathrm{~min}$. After washing with PBS, the fixed cells were stained with DAPI at room temperature in the dark for $5 \mathrm{~min}$. The level of DNA damage was assessed using a laser confocal microscope (A1; Nikon Corporation) at x200 magnification.

Western blot analysis. Cultured gastric cancer cells were collected and the total proteins were extracted using cell lysis buffer (Beyotime Institute of Biotechnology) according to the manufacturer's protocol. The protein concentration of each extract was determined using a Modified Bradford Protein Assay kit (Sangon Biotech Co., Ltd.). Aliquots of total protein (25 $\mu \mathrm{g}$ per lane) were boiled at $100^{\circ} \mathrm{C}$ in loading buffer (cat. no. P0015; Beyotime Institute of Biotechnology), and then separated via $10 \%$ SDS-PAGE. The separated proteins were transferred onto a PVDF membrane (EMD Millipore) that was then blocked with a 5\% lipid-free milk solution for $2 \mathrm{~h}$ at room temperature with gentle rotation. The membrane was incubated with the appropriate primary antibodies diluted in TBST for 1-2 $\mathrm{h}$ at room temperature, after which it was washed three times with TBST, and then incubated with goat anti-rabbit horseradish peroxidase (HRP)-conjugated secondary antibody (dilution, 1:3,000; cat. no. ab6721; Abcam) and goat anti-mouse HRP-conjugated secondary antibody (dilution, 1:3,000; cat. no. ab205719; Abcam) at room temperature for $1 \mathrm{~h}$. The membranes were developed using Pierce ${ }^{\mathrm{TM}}$ ECL Plus Western Blotting Substrate (Pierce; Thermo Fisher Scientific, Inc.) and a total of three independent experiments were performed with GAPDH as the internal standard. The primary antibodies used were as follows: Anti-RAP2A (dilution, 1:1,000; cat. no. ab49685; Abcam), anti-multidrug resistance-associated protein (MRP; dilution, 1:1,000; cat. no. PA5-18315; Thermo Fisher Scientific, Inc.), anti- cleaved caspase-3 (dilution, 1:1,000; cat. no. ab2302; Abcam), and anti-GAPDH (dilution, 1:4,000; cat. no. ab9483; Abcam).

Statistical analysis. All statistical analyses were performed using SPSS Statistics for Windows (version 18.0; SPSS Inc.). Quantitative data are presented as the mean \pm standard deviation. Differences between groups were analyzed using the unpaired Student's t-test or analysis of variance, as appropriate. $\mathrm{P}<0.05$ was considered to indicate a statistically significant result.

\section{Results}

DDP-resistant MGC803/DDP cells exhibit increased viability and migrational capacity. The present study used the MGC803 and corresponding DDP-resistant MGC803/DDP cell lines to investigate the molecular processes that mediate DDP resistance in gastric cancer cells. Both cell lines were treated with a DDP concentration gradient for $24 \mathrm{~h}$, after which viability was assessed using the CCK- 8 method, and the DDP $\mathrm{IC}_{50}$ values were determined. The $\mathrm{IC}_{50}$ values for DDP when treating MGC803 and MGC803/DDP cells were $2.21 \pm 0.13$ and $5.02 \pm 0.10 \mu \mathrm{g} / \mathrm{ml}$, respectively, reflecting a higher level of resistance to DDP in the MGC803/DDP cells $(\mathrm{P}<0.01$; Fig. 1A). Furthermore, following treatment with $1.8 \mu \mathrm{g} / \mathrm{ml}\left(0.8\right.$-fold of $\left.\mathrm{IC}_{50}\right)$ DDP for 24,48 and $72 \mathrm{~h}$ according to the cell growth rate, the MGC803/DDP cells were significantly more viable than the MGC 803 cells, further reflecting their increased resistance to DDP (Fig. 1B). In order to further elucidate the cellular processes associated with DDP tolerance, Transwell matrigel assays were performed to investigate the migration and invasion capabilities of the two cell lines. The results revealed that the migration capability of the MGC803 cells was significantly decreased by DDP $(\mathrm{P}<0.01)$ while the MGC803/DDP cells exhibited no significant change in their ability to migrate following treatment with DDP (Fig. 1C). The matrigel assays demonstrated that the invasive capability of MGC803/DDP cells was relatively insensitive to DDP when compared with that of the MGC803 cells ( $\mathrm{P}<0.01$; Fig. 1D). These results demonstrated that elevated levels of cell viability, and also elevated migration and invasion capabilities, were associated with DDP resistance in gastric cancer cells.

DDP-resistant MGC803/DDP cells exhibit suppressed levels of apoptosis and DNA damage. Apoptotic retardation is a common feature of chemo-resistant cancer cells. In order to test the involvement of apoptotic regulation in the DDP-induced resistance of gastric cancer cells, MGC803 and MGC803/DDP cells were treated with DDP, and their respective apoptotic rates were determined. The results revealed that treatment with DDP significantly increased the apoptotic rate of MGC803 cells, but not MGC803/DDP cells ( $\mathrm{P}<0.01$; Fig. 2A). Hoechst staining was then used to evaluate DNA damage, and it was observed that the degree of DNA damage caused by DDP treatment in MGC803/DDP cells was less than that in MGC803 cells $(\mathrm{P}<0.01$; Fig. 2B). Furthermore, the levels of MRP in the MGC803/DDP cells were higher than those in the MGC803 cells, and no DDP-induced upregulation of cleaved-caspase-3 expression was observed in MGC803/DDP cells; this further confirmed the roles of apoptosis regulation in the induction of DDP resistance. Furthermore, it was also observed that the levels of RAP2A protein in MGC803/DDP cells were higher than those in the MGC803 cells, suggesting an association between RAP2A expression and DDP resistance in gastric cancer cells (Fig. 2C). 

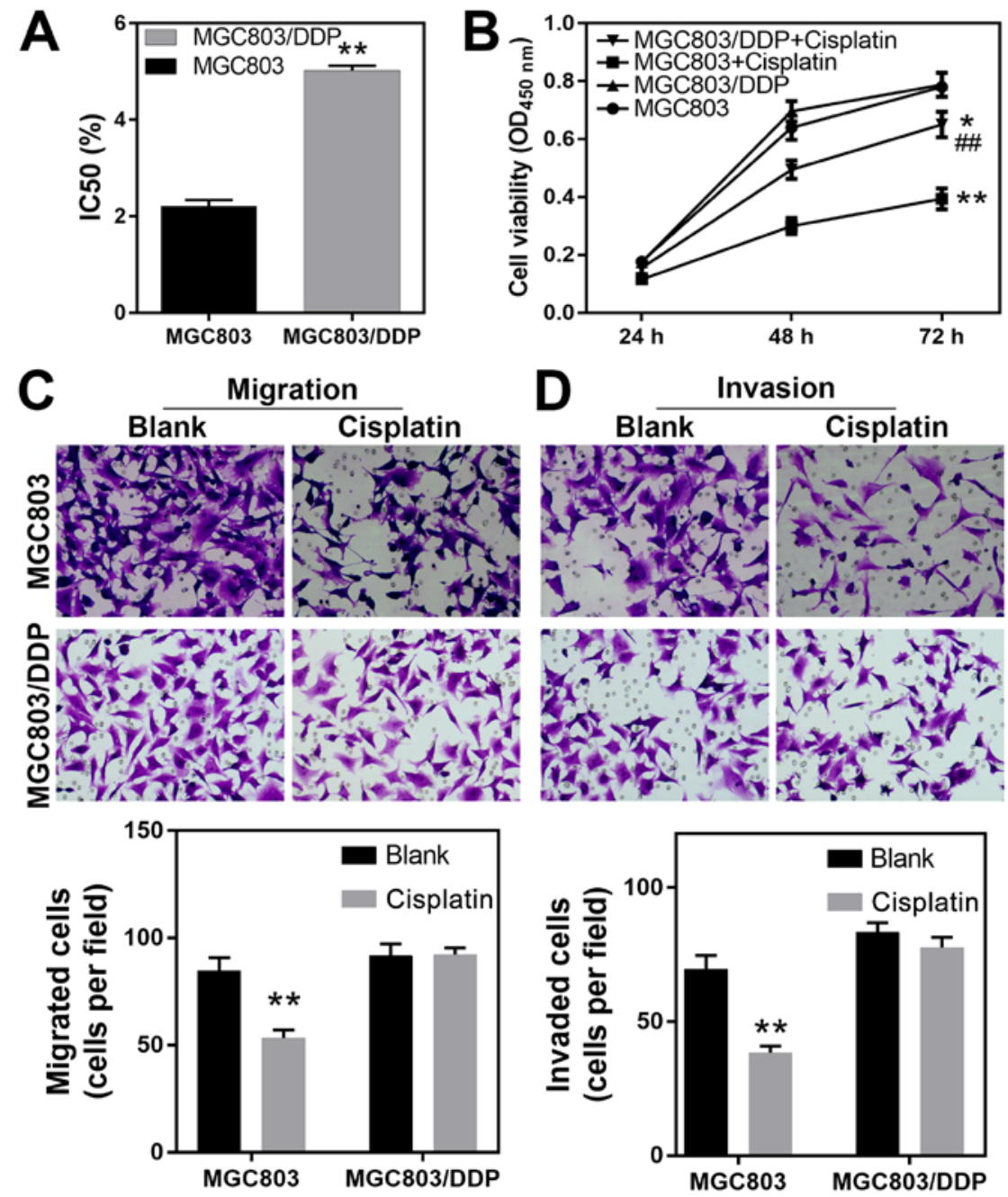

Figure 1. Enhanced viability and invasion capabilities of DDP-resistant gastric cancer cells. (A) $\mathrm{IC}_{50}$ values of DDP when added to MGC803 and MGC803/DDP cells. DDP IC ${ }_{50}$ values were determined by measuring the viability of cells via the CCK-8 method following treatment with a concentration gradient of DDP. (B) Suppression of MGC803 and MGC803/DDP cell viability by DDP treatment. Cells were treated with $1.8 \mu \mathrm{g} / \mathrm{ml}$ DDP for 24,48 and $72 \mathrm{~h}$, and viability was determined using the CCK-8 assay. The (C) migration and (D) invasion capabilities of MGC803 and MGC803/DDP cells were influenced by DDP treatment (x100 magnification). Transwell assays were performed to analyze the migration and invasion capabilities of gastric cancer cells following treatment with $1.8 \mu \mathrm{g} / \mathrm{ml}$ DDP for $24 \mathrm{~h}$. ${ }^{*} \mathrm{P}<0.05,{ }^{* *} \mathrm{P}<0.01$ vs. MGC803 group; ${ }^{\# \#} \mathrm{P}<0.01$ vs. MGC803/DDP group. DDP, cisplatin; $\mathrm{IC}_{50}$, half maximal inhibitory concentration; CCK-8, Cell Counting Kit-8.

RAP2A silencing attenuates DDP resistance in $M G C 8033 / D D P$ cells. In order to investigate the potential role of the RAP2A gene in DDP resistance, siRNA transfection was used to knock down the expression of RAP2A in DDP-resistant MGC8033/DDP cells. siRNA \#1, \#2 and \#3 were used to knock down RAP2A, and the results revealed that siRNA \#3 effectively targeted and blocked the expression of RAP2A (Fig. 3A). Therefore, siRNA \#3 was used for subsequent experimentation. The suppression of RAP2A protein expression in gastric cancer cells was confirmed via western blotting (Fig. 3B and C). The expression levels of MRP were notably decreased by RAP2A siRNA in MGC8033/DDP cells, when compared with those in MGC8033/DDP cells transfected with the NC siRNA (Fig. 3B). By contrast, the expression level of cleaved-caspase-3 in MGC8033/DDP cells was significantly increased by transfection with RAP2A siRNA compared with $\mathrm{DPP}+\mathrm{NC}(\mathrm{P}<0.05$; Fig. 3B and $\mathrm{C})$. The molecular phenotypes induced by RAP2A siRNA also suggested that alterations in cellular function had occurred. It was observed that the viability of DDP-treated MGC8033/DDP cells was decreased by RAP2A knockdown, when compared with the viability of MGC8033/DDP cells treated with the NC siRNA (Fig. 3D). Furthermore, the migrational capability of RAP2A knockdown MGC8033/DDP cells was suppressed following DDP treatment, when compared with that of cells transfected with the NC siRNA (Fig. 3E). Similarly, the invasion capability of DDP-treated MGC8033/DDP cells was also decreased by RAP2A siRNA when compared with that of cells transfected with the NC siRNA $(\mathrm{P}<0.05$; Fig. $3 \mathrm{E})$. These results directly indicate that RAP2A expression in gastric cancer cells promotes the induction of DDP resistance, which may be mediated by the enhanced migration and invasion capabilities of cancerous cells.

RAP2A knockdown promotes MGC803/DDP cell apoptosis during $D D P$ treatment. In order to gain additional information regarding the cellular mechanisms underlying RAP2A-regulated DDP resistance in gastric cancer cells, 


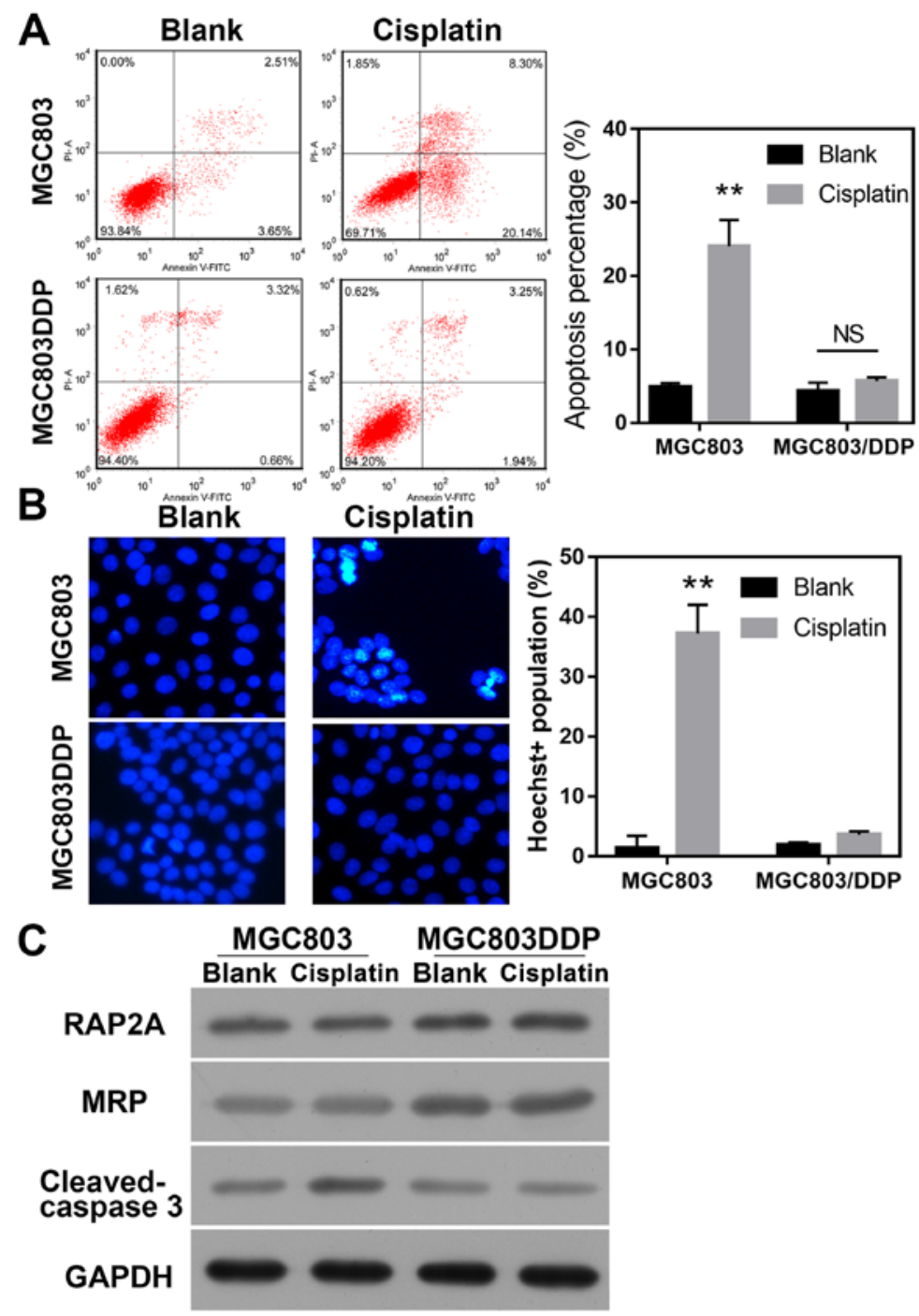

Figure 2. DDP-resistant gastric cancer cells exhibit suppressed levels of apoptosis and DNA damage. (A) Percentages of apoptotic MGC803 and MGC803/DDP cells following DDP treatment. Cell apoptosis following DDP treatment $(1.8 \mu \mathrm{g} / \mathrm{ml}$ for $48 \mathrm{~h}$ ) was evaluated via flow cytometry. (B) DNA damage in MGC803 and MGC803/DDP cells treated with DDP. DNA damage following DDP treatment $(1.8 \mu \mathrm{g} / \mathrm{ml}$ for $48 \mathrm{~h})$ was analyzed by Hoechst 33342 staining and flow cytometry. (C) Levels of RAP2A, MRP and cleaved-caspase-3 in MGC803 and MGC803/DDP cells treated with DDP. Relative levels of protein expression were determined via western blotting, using GAPDH as an internal standard. ${ }^{* * *} \mathrm{P}<0.01$ vs. Blank. DDP, cisplatin; MRP, multidrug resistance-associated protein; NS, not significant; RAP2A, Ras-related protein Rap-2A.

the apoptotic rates of RAP2A knockdown MGC803/DDP cells were further analyzed using flow cytometry. The results revealed that the percentage of apoptotic MGC803/DDP cells during DDP treatment was significantly elevated following RAP2A siRNA transfection, when compared with cells transfected with the NC siRNA ( $\mathrm{P}<0.05$; Fig. 4A). Furthermore, Hoechst 33342 staining revealed that the degree of DNA damage in MGC803/DDP cells under DDP treatment was higher following RAP2A knockdown, which was in contrast to the results of the NC siRNA group $(\mathrm{P}<0.01$; Fig. 4B). These results suggested that RAP2A repressed DDP-induced gastric cancer cell apoptosis and DNA damage, which were both closely associated with the development of resistance to various chemotherapeutic agents, including DDP. Collectively, the present study suggested that RAP2A promoted DDP resistance in gastric cancer cells, potentially by modulating cellular proliferation, migration and invasion, as well as apoptosis and DNA damage during DDP treatment.

\section{Discussion}

Chemotherapy is a major type of cancer treatment that uses a single drug or the combination of multiple agents. It has been widely applied in clinical oncology as a means of curative therapy, or to decrease the symptoms and prolong the survival time of cancer patients $(26,27)$. Chemotherapy has also been administered in combination with other cancer treatments, such as surgery and radiation, or as neoadjuvant or adjuvant therapy given prior to or following other types of cancer treatment (28). However, resistance to chemotherapy is a primary cause of treatment failure, an issue that has become the focus of research in recent decades (29). Previous studies have suggested that specific pump proteins on the surface of cancerous cells (such as p-glycoprotein, which effectively transports chemotherapeutic agents back out of the cell) are critically involved in the resistance to various chemotherapeutic drugs, including DDP $(30,31)$. Further cellular 
A
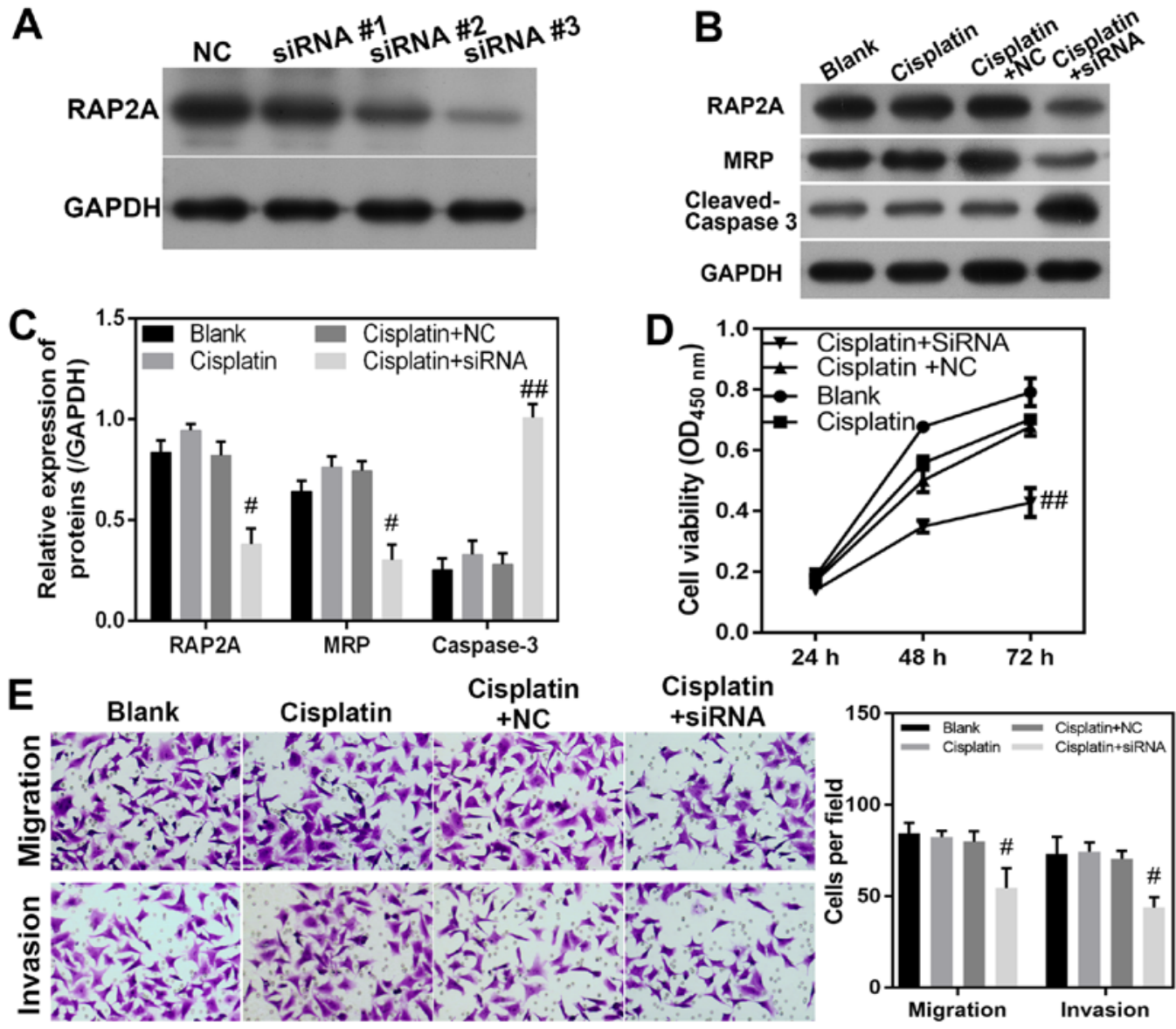

Figure 3. RAP2A promotes migration, invasion and DDP resistance in MGC803/DDP cells. (A) RAP2A expression was knocked down by siRNA (B and C) Levels of RAP2A, MRP and cleaved-caspase-3 in RAP2A-knockdown MGC803/DDP cells during DDP treatment. Relative levels of protein expression were determined via western blotting, with GAPDH serving as an internal standard. (D) Effect of RAP2A siRNA knockdown on MGC803/DDP cell viability during DDP treatment. Cell viability was measured using the Cell Counting Kit-8 assay. (E) Migration and invasion of MGC803/DDP cells with RAP2A siRNA knockdown during DDP treatment for $24 \mathrm{~h}$ (x100 magnification). Cell migration and invasion were analyzed using the Transwell system. Statistical analysis of MGC803/DDP cell migration and invasion. ${ }^{\#} \mathrm{P}<0.05,{ }^{\# \#} \mathrm{P}<0.01$ vs. cisplatin+NC group. RAP2A, Ras-related protein Rap-2a; DDP, cisplatin; siRNA, small interfering RNA; MRP, multidrug resistance-associated protein; NC, negative control; OD, optical density.

and molecular events, including gene amplification, defective cell apoptosis and the promotion of DNA damage repair, also mediate DDP resistance in cancer cells (32-34). Despite recent progress, the underlying molecular mechanisms responsible for the high adaptability of cancer cells and the development of cancer cell resistance to chemotherapeutic agents have only been investigated to a limited extent.

In the present study, DDP resistance in MGC803/DDP cells was confirmed by verifying their increased viability, migration and invasion capabilities, and decreased levels of apoptosis and DNA damage while under treatment with DDP. Subsequent western blot analyses demonstrated increased levels of RAP2A expression in DDP-resistant cells, suggesting that RAP2A may be a GTPase protein able to regulate DDP resistance in gastric cancer cells. Small GTPases, also known as G-proteins, constitute a large group of hydrolase enzymes that hydrolyze GTP to form GDP, and are associated with a number of biological processes, such as cell growth, proliferation, differentiation, movement, lipid vesicle transport and tumorigenesis (35). Notably, numerous members of the G-protein family are reportedly involved in cancer chemotherapy resistance. For example, Rho GTPase enhances the rigidity of ovarian cancer cells and regulates the actin remodeling mechanism to promote resistance to DDP (36). Furthermore, downregulation of RhoB GTPase expression levels in laryngeal carcinoma cells was demonstrated to promote the development of acquired DDP resistance (36). Finally, Rho GDP dissociation inhibitor 2, a regulator of the Rho family of GTPases, contributes to DDP resistance in gastric cancer cells by enhancing the expression of the Bcl-2 gene (37). GTPases and their activating proteins, such as the $76 \mathrm{kDa}$ Ral-binding GTPase activating protein, are involved in the development of resistance to multiple chemotherapeutic drugs (including vinorelbine, doxorubicin and DDP) via their ability to transport these drugs back out of cancerous cells (38-40). Furthermore, autophagy and other cellular processes regulated by GTPase members such as the Rac3 GTPase $(41,42)$, were demonstrated to mediate the development of cancer cell resistance to antitumor drugs such as DDP $(43,44)$. As a GTPase family member, the significant increase of RAP2A expression levels in MGC803/DDP cells suggested that RAP2 contributes to the resistance of gastric cancer cells to DDP treatment.

To test this hypothesis, the present study knocked down RAP2A in DDP-resistant MGC803/DDP cells via transfection with specific siRNAs. In addition to the subsequent decrease in RAP2A expression level, the viability and invasion capabilities 

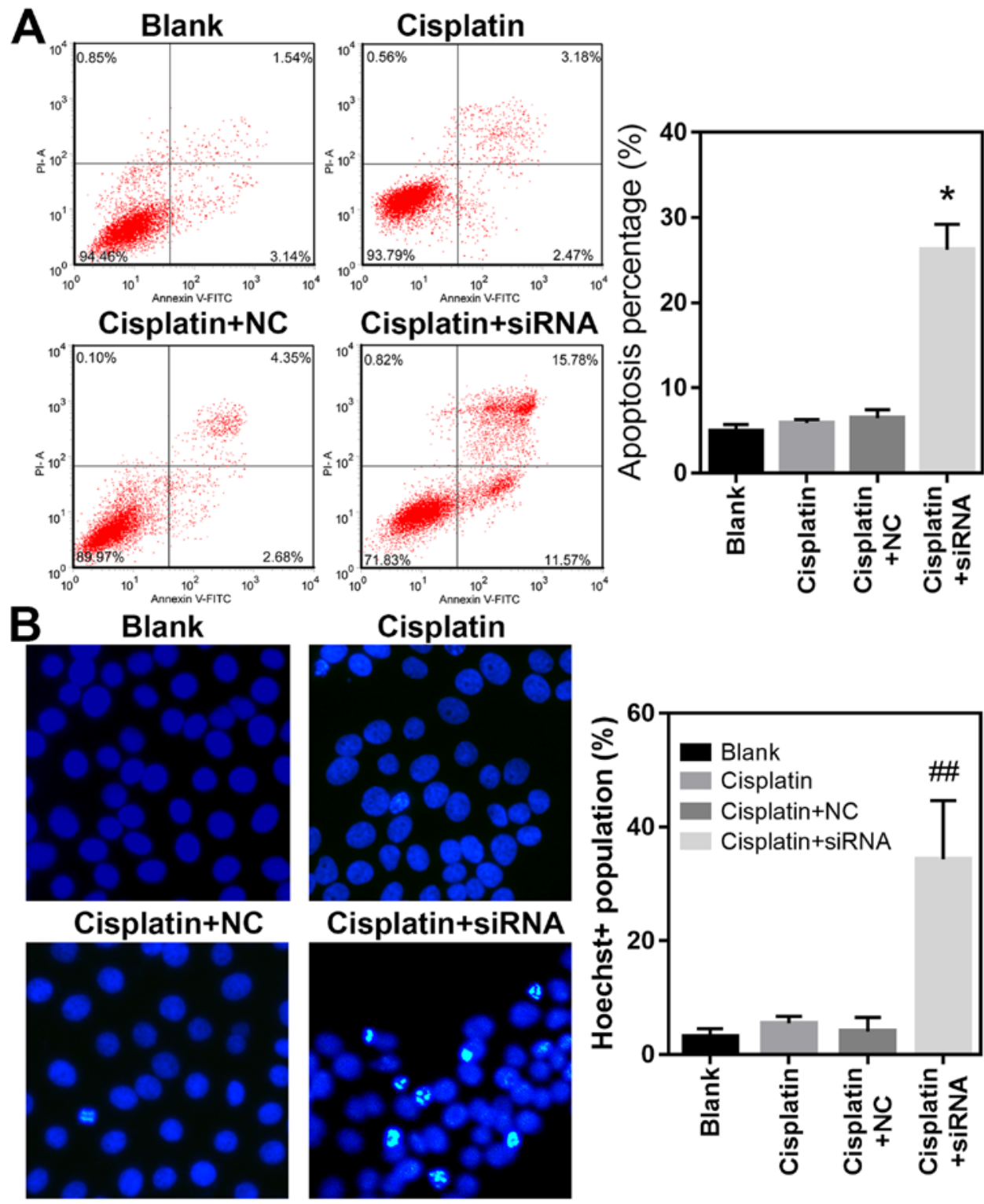

Figure 4. RAP2A inhibits apoptosis and DNA damage in MGC803/DDP cells. (A) Percentages of apoptotic MGC803/DDP cells with RAP2A siRNA knockdown following DDP treatment. Cell apoptosis following DDP treatment was analyzed via flow cytometry. (B) DNA damage in MGC803/DDP cells with RAP2A siRNA knockdown during DDP treatment. DNA damage was evaluated via Hoechst 33342 staining and flow cytometry. ${ }^{*} \mathrm{P}<0.05,{ }^{\# \#} \mathrm{P}<0.01$ vs. cisplatin+NC group. RAP2A, Ras-related protein Rap-2a; DDP, cisplatin; siRNA, small interfering RNA; NC, negative control.

of the MGC803/DDP cells were also suppressed by RAP2A siRNA under DDP treatment. The inhibition of DDP resistance in RAP2A-knockdown MGC803/DDP cells was also confirmed by increases in cell apoptosis and DNA damage. The cellular analysis of DDP-resistant cells demonstrated that RAP2A may be a positive regulator of DDP resistance in gastric cancer cells. A previous study demonstrated that p53 was able promote RAP2A expression in cancer cells, and that this further activated the matrix metalloproteinase (MMP) enzymes MMP2 and MMP9 via phosphorylation of AKT (22). In addition, the RAP2A protein can be specifically ubiquitinated by E3 ubiquitin ligase neuronal precursor cell expressed and developmentally downregulated protein 4-1, and subsequently contributes to the increased migration and invasion capabilities of glioma cells (45). These mechanisms that enable RAP2A to regulate tumor development may also mediate gastric cancer progression and the development of
DDP resistance, and therefore deserve further investigation. Additional investigation into the roles of RAP2A expression in the resistance to other chemotherapeutic drugs may also broaden the current understanding of cancer chemotherapy resistance.

In summary, the present study demonstrated that RAP2A gene expression was increased in DDP-resistant gastric cancer cells. This enhanced level of RAP2A expression promoted gastric cancer cell resistance to DDP by regulating cell viability, migration, invasion, apoptosis and DNA damage. These findings provide novel insights into the molecular mechanisms underlying DDP resistance in gastric cancer, as well as the pathogenic roles played by GTPase proteins during the development of chemotherapy resistance. However, the present study was not performed in vivo, and further studies are required in order to demonstrate the effects of RAB2P expression on drug resistance in gastric cancer. 


\section{Acknowledgements}

Not applicable.

\section{Funding}

The present study was supported by the Science and Technology Program of Huzhou, Applied Research for Public Welfare (grant no. 2017GYB40) and Zhejiang Medical and Health Science and Technology Plan Project (grant no. 2019ZD050).

\section{Availability of data and materials}

The datasets used and/or analyzed during the current study are available from the corresponding author on reasonable request.

\section{Authors' contributions}

JZ and JM designed the experiments. JZ performed the experiments and drafted the manuscript. YWe and HS were involved in flow cytometry experiments. JZ, YWa, LY and GC collected the data and performed data analysis. JM revised the manuscript. All authors approved the final version before submission.

\section{Ethics approval and consent to participate}

Not applicable.

\section{Patient consent for publication}

Not applicable.

\section{Competing interests}

The authors declare that they have no competing interests.

\section{References}

1. Stewart BW, Wild CP (eds.): World Cancer Report 2014. IARC, Lyon, France, pp 839-851, 2014.

2. Torre LA, Siegel RL, Ward EM and Jemal A: Global cancer incidence and mortality rates and trends-an update. Cancer Epidemiol Biomarkers Prev 25: 16-27, 2016.

3. Kamran SC, Hong TS and Wo JY: Advances in the management of gastric and gastroesophageal cancers. Curr Oncol Rep 18: 13, 2016.

4. Lee YY and Derakhshan MH: Environmental and lifestyle risk factors of gastric cancer. Arch Iran Med 16: 358-365, 2013.

5. Wang K, Yuen ST, Xu J, Lee SP, Yan HH, Shi ST, Siu HC Deng S, Chu KM, Law S, et al: Whole-genome sequencing and comprehensive molecular profiling identify new driver mutations in gastric cancer. Nat Genet 46: 573-582, 2014

6. Venerito M, Vasapolli R, Rokkas T, Delchier JC and Malfertheiner P: Helicobacter pylori, gastric cancer and other gastrointestinal malignancies. Helicobacter 22 (Suppl 1), 2017.

7. Yoon $\mathrm{H}$ and Kim N: Diagnosis and management of high risk group for gastric cancer. Gut Liver 9: 5-17, 2015.

8. Aurello P, Sagnotta A, Terrenato I, Berardi G, Nigri G, D'Angelo F and Ramacciato G: Oncologic value of laparoscopy-assisted distal gastrectomy for advanced gastric cancer: A systematic review and meta-analysis. J Minim Access Surg 12: 199-208, 2016

9. Chen K, Xu XW, Zhang RC, Pan Y, Wu D and Mou YP: Systematic review and meta-analysis of laparoscopy-assisted and open total gastrectomy for gastric cancer. World $\mathrm{J}$ Gastroenterol 19: 5365-5376, 2013.
10. Janunger KG, Hafström L and Glimelius B: Chemotherapy in gastric cancer: A review and updated meta-analysis. Eur J Surg 168: 597-608, 2002.

11. Othman MO and Wallace MB: Endoscopic mucosal resection (EMR) and endoscopic submucosal dissection (ESD) in 2011, a Western perspective. Clin Res Hepatol Gastroenterol 35: 288-294, 2011.

12. Liang L, Fang JY and Xu J: Gastric cancer and gene copy number variation: Emerging cancer drivers for targeted therapy. Oncogene 35: 1475-1482, 2016.

13. Valentini V and Cellini F: Radiotherapy in gastric cancer: A systematic review of literature and new perspectives. Expert Rev Anticancer Ther 7: 1379-1393, 2007.

14. Wagner AD, Unverzagt S, Grothe W, Kleber G, Grothey A, Haerting $J$ and Fleig WE: Chemotherapy for advanced gastric cancer. Cochrane Database Syst Rev: CD004064, 2010.

15. Stordal B and Davey M: Understanding cisplatin resistance using cellular models. IUBMB Life 59: 696-699, 2007.

16. Jacobsen $\mathrm{C}$ and Honecker F: Cisplatin resistance in germ cell tumours: Models and mechanisms. Andrology 3: 111-121, 2015.

17. Yang SM, Huang C, Li XF, Yu MZ, He Y and Li J: miR-21 confers cisplatin resistance in gastric cancer cells by regulating PTEN. Toxicology 306: 162-168, 2013.

18. Sun XP, Dong X, Lin L, Jiang X, Wei Z, Zhai B, Sun B, Zhang Q, Wang X, Jiang $\mathrm{H}$, et al: Up-regulation of survivin by AKT and hypoxia-inducible factor $1 \alpha$ contributes to cisplatin resistance in gastric cancer. FEBS J 281: 115-128, 2014.

19. Yang M, Shan X, Zhou X, Qiu T, Zhu W, Ding Y, Shu Y and Liu P: miR-1271 regulates cisplatin resistance of human gastric cancer cell lines by targeting IGF1R, IRS1, mTOR, and BCL2. Anticancer Agents Med Chem 14: 884-891, 2014.

20. Xu W, Wang S, Chen Q, Zhang Y, Ni P, Wu X, Zhang J, Qiang F, Li A, Røe OD, et al: TXNL1-XRCC1 pathway regulates cisplatin-induced cell death and contributes to resistance in human gastric cancer. Cell Death Dis 5: e1055, 2014.

21. Gloerich M, ten Klooster JP, Vliem MJ, Koorman T, Zwartkruis FJ, Clevers H and Bos JL: Rap2A links intestinal cell polarity to brush border formation. Nat Cell Biol 14: 793-801, 2012.

22. Wu JX, Zhang DG, Zheng JN and Pei DS: Rap2a is a novel target gene of $\mathrm{p} 53$ and regulates cancer cell migration and invasion. Cell Signal 27: 1198-1207, 2015.

23. Huang D, Duan H, Huang H, Tong X, Han Y, Ru G, Qu L, Shou C and Zhao Z: Cisplatin resistance in gastric cancer cells is associated with HER2 upregulation-induced epithelial-mesenchymal transition. Sci Rep 6: 20502, 2016.

24. Kramer N, Walzl A, Unger C, Rosner M, Krupitza G, Hengstschläger $\mathrm{M}$ and Dolznig $\mathrm{H}$ : In vitro cell migration and invasion assays. Mutat Res 752: 10-24, 2013.

25. Erba E, Ubezio P, Broggini M, Ponti M and D'Incalci M: DNA damage, cytotoxic effect and cell-cycle perturbation of Hoechst 33342 on L1210 cells in vitro. Cytometry 9: 1-6, 1988.

26. Redondo-Blanco S, Fernández J, Gutiérrez-Del-Río I, Villar CJ and Lombó F: New insights toward colorectal cancer chemotherapy using natural bioactive compounds. Front Pharmacol 8: $109,2017$.

27. Zitvogel L, Apetoh L, Ghiringhelli F and Kroemer G: Immunological aspects of cancer chemotherapy. Nat Rev Immunol 8: 59-73, 2008.

28. Meeks JJ, Bellmunt J, Bochner BH, Clarke NW, Daneshmand S, Galsky MD, Hahn NM, Lerner SP, Mason M, Powles T, et al: A systematic review of neoadjuvant and adjuvant chemotherapy for muscle-invasive bladder cancer. Eur Urol 62: 523-533, 2012.

29. Sui X, Chen R, Wang Z, Huang Z, Kong N, Zhang M, Han W, Lou F, Yang J, Zhang Q, et al: Autophagy and chemotherapy resistance: A promising therapeutic target for cancer treatment. Cell Death Dis 4: e838, 2013

30. Terek RM, Schwartz GK, Devaney K, Glantz L, Mak S, Healey JH and Albino AP: Chemotherapy and P-glycoprotein expression in chondrosarcoma. J Orthop Res 16: 585-590, 1998.

31. Gibalová L, Sereš M, Rusnák A, Ditte P, Labudová M, Uhrík B, Pastorek J, Sedlák J, Breier A and Sulová Z: P-glycoprotein depresses cisplatin sensitivity in L1210 cells by inhibiting cisplatin-induced caspase-3 activation. Toxicol In Vitro 26: 435-444, 2012.

32. Liu LZ, Zhou XD, Qian G, Shi X, Fang J and Jiang BH: AKT1 amplification regulates cisplatin resistance in human lung cancer cells through the mammalian target of rapamycin/p70S6K1 pathway. Cancer Res 67: 6325-6332, 2007. 
33. Bauer JA, Kumar B, Cordell KG, Prince ME, Tran HH, Wolf GT, Chepeha DB, Teknos TN, Wang S, Eisbruch A, et al: Targeting apoptosis to overcome cisplatin resistance: A translational study in head and neck cancer. Int J Radiat Oncol Biol Phys 69 (2 Suppl): S106-S108, 2007.

34. Yu WK, Wang Z, Fong CC, Liu D, Yip TC, Au SK, Zhu G and Yang $M$ : Chemoresistant lung cancer stem cells display high DNA repair capability to remove cisplatin-induced DNA damage. Br J Pharmacol 174: 302-313, 2017.

35. Okada T, Sinha S, Esposito I, Schiavon G, López-Lago MA, Su W, Pratilas CA, Abele C, Hernandez JM, Ohara M, et al: The Rho GTPase Rnd1 suppresses mammary tumorigenesis and EMT by restraining Ras-MAPK signalling. Nat Cell Biol 17: 81-94, 2015.

36. Sharma S, Santiskulvong C, Rao J, Gimzewski JK and Dorigo O: The role of Rho GTPase in cell stiffness and cisplatin resistance in ovarian cancer cells. Integr Biol (Camb) 6: 611-617, 2014.

37. Cho HJ, Baek KE, Park SM, Kim IK, Nam IK, Choi YL, Park SH, Im MJ, Choi J, Ryu J, et al: RhoGDI2 confers gastric cancer cells resistance against cisplatin-induced apoptosis by upregulation of Bcl-2 expression. Cancer Lett 311: 48-56, 2011.

38. Awasthi S, Sharma R, Yang Y, Singhal SS, Pikula S, Bandorowicz-Pikula J, Singh SV, Zimniak P and Awasthi YC: Transport functions and physiological significance of $76 \mathrm{kDa}$ Ral-binding GTPase activating protein (RLIP76). Acta Biochim Pol 49: 855-867, 2002.

39. Stuckler D, Singhal J, Singhal SS, Yadav S, Awasthi YC and Awasthi S: RLIP76 transports vinorelbine and mediates drug resistance in non-small cell lung cancer. Cancer Res 65: 991-998, 2005 .
40. Vatsyayan R, Chaudhary P, Lelsani PC, Singhal P, Awasthi YC, Awasthi S and Singhal SS: Role of RLIP76 in doxorubicin resistance in lung cancer. Int J Oncol 34: 1505-1511, 2009.

41. Wu R, Murali R, Kabe Y, French SW, Chiang YM, Liu S, Sher L, Wang CC, Louie S and Tsukamoto H: Baicalein targets GTPase-mediated autophagy to eliminate liver tumor initiating stem cell-like cells resistant to mTORC1 inhibition. Hepatology 68: 1726-1740, 2018.

42. Zhu WL, Hossain MS, Guo DY, Liu S, Tong H, Khakpoor A, Casey PJ and Wang M: A role for Rac3 GTPase in the regulation of autophagy. J Biol Chem 286: 35291-35298, 2011.

43. Sirichanchuen B, Pengsuparp T and Chanvorachote P: Long-term cisplatin exposure impairs autophagy and causes cisplatin resistance in human lung cancer cells. Mol Cell Biochem 364: 11-18, 2012.

44. Kumar P, Zhang DM, Degenhardt K and Chen ZS: Autophagy and transporter-based multi-drug resistance. Cells 1: 558-575, 2012.

45. Wang L, Zhu B, Wang S, Wu Y, Zhan W, Xie S, Shi H and Yu R: Regulation of glioma migration and invasion via modification of Rap2a activity by the ubiquitin ligase Nedd4-1. Oncol Rep 37: 2565-2574, 2017. International (CC BY-NC-ND 4.0) License. 